\title{
面向工业监控应用的广域异构无线网络端到端 时延分析
}

\author{
王金涛 ${ }^{(12)} ，$ 金䀬 $^{(1)} ，$ 曾鹏 $^{(1 *} ，$ 李栋 $^{(12)}$ \\ (1) 中国科学院网络化控制系统重点实验室, 中国科学院沈阳自动化研究所, 沈阳 110016 \\ (2) 中国科学院大学, 北京 100049 \\ * 通信作者. E-mail: zp@sia.cn
}

收稿日期: 2015-05-26; 接受日期: 2015-06-23; 网络出版日期: 2015-10-12 中国科学院战略性科技先导专项 (批准号: XDA06020500) 资助

\begin{abstract}
摘要 随着无线通信技术的发展, 基于 WirelessHART, WIA-PA 和 ZigBee 等协议的无线技术已广泛 应用于工业控制领域中. 但由于资金、技术、设备等条件的限制, 在一定时期内会存在多种网络技术 并存的情况. 时延分析是一种在网络设计阶段检测网络实时性的有效手段, 而目前没有针对工业广 域异构网络进行端到端时延分析的研究. 因此, 本文以面向工业检测和控制应用的广域异构回程网 为依托, 对基于 IEEE 802.11 构建的长距离 WIFI, Wireless HART, ZigBee 和 WIA-PA 网络共存环境下 的广域异构网络的端到端时延进行理论分析与实验验证. 实验结果表明, 当 802.11 长距离 WIFI 回 程网节点数目大于 25 , 网关节点数据聚合周期小于 $30 \mathrm{~ms}$ 时, 包含 ZigBee 子网的广域异构网络时延 悲观率在 2.3 以内, 包含其他子网的广域异构网络时延悲观率在 1.5 以内, 根据已有的研究分析表明 本文的方法具有合理性和有效性.
\end{abstract}

关键词工业监控 异构网 无线网络 端到端时延

\section{1 引言}

由于无线传输技术具有成本低、维护方便、灵活性好等优点, 因此被越来越多地应用于工业控制 网络中 ${ }^{[1,2]}$. 在现代化的工业生产中, 工厂与工厂之间, 以及同一工厂的不同园区之间数据的交互愈发 频繁.但由于在工业控制网络的发展过程中, 不同企业甚至同一企业在不同时期、不同厂区都会选择基 于不同标准的工业控制系统, 因此形成了异构的工业控制网络. 网络的异构性是影响数据传输质量的 重要因素. WirelessHART 和 ZigBee 都是无线工业控制领域应用广泛的过程检测与控制标准, WIA-PA 则是由中国科学院沈阳自动化研究所主导推出的, 具有自主知识产权的高可靠、超低功耗的智能多跳 无线传感器网络国家标准. 在众多行业标准并行的情况下, 对使用不同标准的异构网络进行综合分析 显得十分必要.

时延分析是系统设计阶段用于获得数据从发送节点到目的节点 (简称为端到端) 所用时间的一类 方法, 该类方法能发现错误的系统设计所引发的问题, 例如时延敏感的控制数据因传输时间过长而错 过有效的控制时机导致生产失败甚至生命财产损失. 在对网络实时性分析方法的合理性与有效性界定 
方面, 研究者通常根据时延的分析结果与在实际网络中的差异进行比较来判断分析方法的优劣. 本文 依托中国科学院战略性先导科技专项 一一面向感知中国的新一代信息技术研究, 以工业监控 (监测 和控制) 应用为研究背景, 以工业生产中的异构网络为例, 分析在基于 IEEE 802.11 构建的由长距离 WIFI ${ }^{[3]}$, WirelessHART ${ }^{1)}$, ZigBee ${ }^{2)}$ 和 WIA-PA ${ }^{[4]}$ 组成的异构网络环境中数据的端到端时延.

由于在工业监控网中传送的数据包含工业现场大量实时的、非实时的管理控制和图形、图像信息 (据统计, 在一套现代工业设施中安装的传感器数量可达 30000 个之多) ${ }^{[5]}$, 因此在一个广域异构网络 中将同时存在大量的数据. 在庞大的数据量下, 加之网络的异构性影响, 使得对于工业监控数据的时 延分析变得更加困难. 虽然通过系统仿真也可获得数据的端到端时延, 但对于本文所关注的广域异构 网络, 系统仿真不能覆盖系统所有的状态空间, 并且其运行所需时间也是无法接受的.

本文主要对 IEEE 802.11 长距离 WIFI 及 WIA-PA 网络的端到端时延进行了系统的分析, 然后以 工业异构监控网络应用为背景, 建立起一套包含 WirelessHART, Zigbee, WIA-PA 和长距离 WIFI 的 异构网络端到端时延分析架构. 为时延敏感型数据的及时、可靠传输提供设计保障.

研究中以如图 1 所示的一种工业生产中的典型异构网络为例, 分析数据通过该异构网络的端到 端时延. 工业生产过程中的数据类型可以分为数字、图形、图像三种 ${ }^{[6]}$. 数字信息包括传感信息、启 停控制信息等. 图形信息的主要特点是在不同时段的数据类型和格式可能不同, 可以是电信号, 也可 以是数字. 图像信息的来源则是对工业现场站、库的实时视频监控. 图 1 中包含 IEEE 802.11 长距离 WIFI, WirelessHART, ZigBee 和 WIA-PA 四种网络形式. WirelessHART, ZigBee 和 WIA-PA 负责传 感数据的采集与传输, 最后通过 IEEE 802.11 长距离 WIFI 回程网传输到数据的存储与控制中心.

\section{2 相关工作}

在工业异构网络中, 如何进行数据的端到端时延分析是一个关键的研究问题. 现有的研究中, 对 于端到端时延的定量分析主要集中于对单个网络类型的分析, 而对异构网络整体的端到端时延分析的 研究还较少, 现将相关研究归纳总结如下.

\section{1 异构网络的端到端时延分析}

文献 [7] 研究了 CAN 总线和工业以太网融合的异构网络的时延. 文献 [8 10] 采用网络演算的方 法来分析异构网络的端到端时延, 但是由于网络演算本身存在计算量与计算误差较大的问题, 无法精 确对异构网络的时延进行分析. 文献 [11 14] 采用帧轨迹 (trajectory approach) 的方法来求解交换式 以太网、AFDX (avionics full DupleX switched ethernet)、Ad hoc 等组成的异构网络的时延上界, 但是 以上的分析均面向有线控制网络的场景, 在无线工业控制领域由于网络特性的不同而不能完全适用.

\section{2 单一类型工业无线网络的时延分析}

对各种单一网络类型的时延分析中, 在 WirelessHART 网络的分析方面, 文献 $[15,16]$ 将 WirelessHART 网络的端到端时延分析问题映射成多核调度问题, 通过对最差执行时间的分析, 得到基于固 定优先级调度的 WirelessHART 网络实时数据流的最差执行时间. 文献 [17] 得到了一种传感网中的端 到端时延界限, 但是只是针对树状拓扑、单信道和簇内时分机制. 文献 [18] 提出了针对传感网的网络 演算方法, 通过计算得到了数据在最差情况下的时延.

1) WirelessHART specification. http://www.hartcomm2.org. 2007.

2) ZigBee specification. http://www.zigbee.org/Standards/Overview.aspx. 2014. 


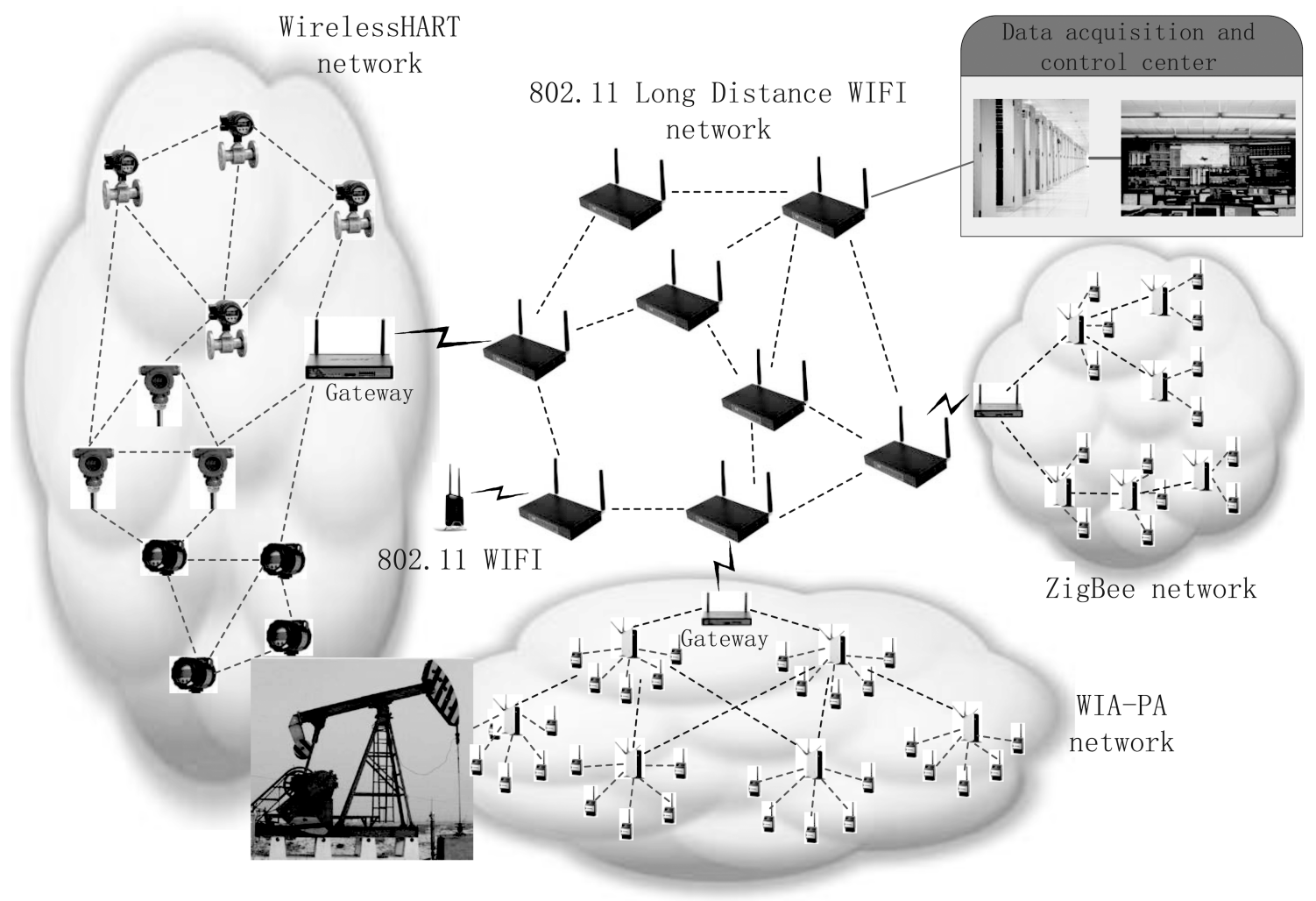

图 1 工业异构控制网络

Figure 1 Industrial heterogeneous control network

在对 ZigBee 网络的时延分析方面, 文献 [19] 分析了 ZigBee 网络在工业自动化领域针对实时性的 应用, 其目标是验证该协议是否能够满足工业自动化领域的所有需求. 并且面向实时性应用对该协议 的性能进行了理论分析. 文献 [20] 提出了用于医疗中的人体多跳异构网络, 对其端到端时延进行了分 析, 但是其网络模式包括 WLAN, WiMAX, UMTS 和 ZigBee 四种形式, 不适用于工业应用领域.

在 WIA-PA 的时延分析方面, 文献 [21] 分析了可预期周期性数据和不可预期数据在不同场景下 的时延. 文献 [22] 提出了一种面向多信道 WIA-PA 的分布式动态调度机制, 可以减少计算时间、最大 化网络利用率、减少重传次数, 从而提高实时性, 但是没有进行系统地数学分析.

在对 802.11 长距离 WIFI 网络的时延分析方面, 由于本文采用的是基于 $2 \mathrm{P}$ MAC 的特殊传输模 式, 因此尚未在现有的研究成果中找到针对此种网络形式进行端到端时延分析的文章.

综上, 由于无线网络的异构性, 存在链路接入方法、传输调度机制和信道使用规则等多方面的差 异, 传统的只针对单个网络的分析方法无法完成异构环境下工业控制网络的时延分析, 而已有的异构 通信媒介的相关工作没有包含工业无线网络协议的研究.

\section{3 工业监控异构网络模型}

\section{1 网络模型}

工业监控网络是指由协调器、路由器和终端节点设备等组成的, 用于工业领域对网内计算机及工 
业设备进行监测和控制的网络. 其中监测类数据主要是图形、图像信息, 具有高带宽、低实时性需求, 而控制类数据则主要是具有低带宽、高实时性需求的工业控制信息.

本文面向如图 1 所示的异构工业监控网络, 将各厂区已使用的无线网络 (包括 WirelessHART, WIA-PA 和 ZigBee ) 作为异构网络的各个子网, 各子网间通过由 IEEE802.11 长距离 WIFI 实现的工 业回程网相连, 构成广域异构工业监控网络. 网络中各子网将采集到的数据通过各自的网关传入回程 网, 经回程网传输到数据中心供总控中心使用. 从总控中心发出的控制命令依次通过回程网, 子网网 关到达子网中的受控设备. 在该网络中, 高带宽、低时延需求的监测图形、图像数据流和低带宽高实 时性需求的工业控制数据流同时存在.

将异构网络采用二元组 $G=\langle V, E\rangle$ 表示. 网络中包括传感器、路由器、网关等不同类型的节点, 用 $V=\left\{v_{1}^{L D}, v_{2}^{L D}, \ldots, v_{n_{L D}}^{L D} ; v_{1}^{Z B}, v_{2}^{Z B}, \ldots, v_{n_{Z} B}^{Z B} ; v_{1}^{W H}, v_{2}{ }^{W H}, \ldots, v_{n_{W H}}^{W H} ; v_{1}^{W P}, v_{2}{ }^{W P}, \ldots, v_{n_{W P}}^{W P}\right\}$ 来表 示这些不同的设备. $v_{1}^{L D}, v_{2}{ }^{L D}, \ldots, v_{n_{L D}}^{L D}$ 表示 802.11 长距离 WIFI 中的节点, $v_{1}^{Z B}, v_{2}{ }^{Z B}, \ldots, v_{n_{Z B}}^{Z B}$ 表 示 ZigBee 中的节点, $v_{1}^{W H}, v_{2}{ }^{W H}, \ldots, v_{n_{W H} W}^{W H}$ 表示 WirelessHART 中的节点, $v_{1}^{W P}, v_{2}{ }^{W P}, \ldots, v_{n_{W P} P}$ 表示 WIA-PA 中的节点. $n_{L D}, n_{Z B}, n_{W H}, n_{W P}$ 分别表示相应网络节点数, 并有 $n_{L D}+n_{Z B}+n_{W H}+n_{W P}=$ $n . E$ 是网络中链路的集合, $E$ 中的每个元素表示节点之间的一条可用的链路.

用 $F$ 代表网络中的数据流集合, 每条数据流 $F_{k}$ 通过三元组 $F_{k}=\left\langle t_{k}, d_{k}, R_{k}\right\rangle$ 表示. $t_{k}$ 代表数据 流中的数据发送周期, $d_{k}$ 表示数据流 $F_{k}$ 从源节点到目的节点所经历的总跳数. 相应地在 802.11 长距 离 WIFI, ZigBee, WirelessHART 和 WIA-PA 网络中每种网络经过的跳数分别记为 $d_{k}^{L D}, d_{k}^{Z B}, d_{k}^{W H}$ 和 $d_{k}^{W P} . R_{k}$ 表示数据流 $F_{k}$ 所经历的端到端时延上界. 对 4 种网络的端到端时延分别记为 $R_{k}^{L D}, R_{k}^{Z B}$, $R_{k}^{W H}$ 和 $R_{k}^{W P}$.

\section{2 问题描述}

本文研究广域异构网络中工业控制类数据的端到端时延. 广域异构网络端到端时延主要是指, 对 于高实时性需求的工业控制类数据, 从各个子网 (包括 ZigBee, WirelessHART 和 WIA-PA) 节点到数 据中心节点所经历的时延大小. 由于网络的时延与网络负载有关, 因此在对各子网进行时延分析时对 负载进行考虑. 但是在长距离 WIFI 回程网中, 由于高实时性控制类数据具有比监测类数据更高的优 先级, 而控制类数据属于低带宽需求的业务, 因此对于该类业务, 在长距离 WIFI 回程网中认为其带宽 是足够的, 所以进行整个网络的端到端传输时延分析时, 不再专门考虑负载的影响.

工业控制类数据从子网的传感器节点出发, 经过子网传输到网关节点, 由网关节点进行聚合之后, 经 WIFI 回程网传输到数据采集与控制中心. 因此, 数据所经历的端到端时延由 3 部分组成: 子网的时 延、WIFI 回程网的时延和网关节点处数据聚合的时延. 由于各子网中的传感器节点数量多, 但每个节 点采集到的数据量较少, 因此在各子网内将多个传感器节点的小数据包合并为一个较大的数据包后再 传输到回程网中, 此过程称为聚合. 进行数据聚合可以有效降低网络传输的总数据量, 减小数据冲突的 概率, 减轻网络拥塞, 有效节省能源开销, 达到延长网络寿命的效果. 在 IEEE 802.11 长距离 WIFI 与 其他网络连接的网关节点处要进行数据聚合. 本文中将聚合周期用 $T_{a g}$ 表示, 由数据聚合引起的时延 为 $R_{a g}=T_{a g}$. 因此, 数据由终端节点到数据中心的总时延为 $R_{k}=R_{k}^{L D}+R_{k}^{x}+R_{a g}=R_{k}^{L D}+R_{k}^{x}+T_{a g}$ 其中 $R_{k}^{x} \in\left\{R_{k}^{Z B}, R_{k}^{W H}, R_{k}^{W P}\right\}$.

\section{4 网络的端到端时延分析}

工业广域异构网络包括多种网络形式, 因此本节分别分析各网络中的端到端时延, 再对整体网络 
进行分析.

\subsection{WirelessHART 网络的时延分析}

本文采用的 WirelessHART 网络时延分析方法为 EDA ${ }^{[15]}$ 分析, 该方法是目前最先进的针对 WirelessHART 网络中固定优先级调度策略的数据端到端时延分析方法.

WirelessHART 网络的时延分析包括两步, 第 1 步计算由于信道竞争产生的时延, 得到最差情况 下的端到端时延上界 (因为未包括冲突造成的时延, 所以将其称为伪上界), 用 $R_{k}^{W H-c h}$ 表示. 第 2 步 再将由于传输冲突造成的时延加入到第 1 步得到的时延中, 得到真正的端到端时延上界.

定义1 (k- 繁忙期) 在数据流 $F_{k}$ 完成其数据包传输之前, 节点的所有信道被比 $F_{k}$ 优先级高的 数据流所占据的最大连续时间称为 $\mathrm{k}$ - 繁忙期. 比 $F_{k}$ 优先级高的数据流的集合用 $\mathrm{hp}\left(F_{k}\right)$ 表示.

定义 2 (插入负载) 对于一个数据流 $F_{i}, F_{i} \in \mathrm{hp}\left(F_{k}\right)$, 如果存在数据包的生成时间 (release time) 比 $\mathrm{k}$ - 忙碌期早, 而其截止期在 $\mathrm{k}$ - 忙碌期内, 则称在数据流 $F_{k}$ 的 $\mathrm{k}$ - 忙碌期中具有插入负载 (carry-in workload).

在 $\alpha$ 时间内, 在无插入负载情况下和存在插入负载情况下, 周期为 $t_{i}$ 的数据流 $F_{i}$ 对 $F_{k}$ 产生的 两种干扰分别为

$$
\begin{aligned}
& I_{k}^{N C}\left(F_{i}, \alpha\right)=\min \left(\left\lfloor\frac{\alpha}{t_{i}}\right\rfloor \cdot c_{i}+\min \left(\alpha \bmod t_{i}, c_{i}\right), \alpha-c_{k}+1\right), \\
& I_{k}^{C I}\left(F_{i}, \alpha\right)=\min \left(\left\lfloor\frac{\max \left(\alpha-c_{i}, 0\right)}{t_{i}}\right\rfloor \cdot c_{i}+c_{i}+\mu_{i}, \alpha-c_{k}+1\right),
\end{aligned}
$$

其中, $\mu_{i}=\min \left(\max \left(\max \left(\alpha-c_{i}, 0\right)-\left(t_{i}-R_{i}\right), 0\right), c_{i}-1\right), R_{i}$ 表示数据流 $F_{i}$ 的最大端到端时延.

由于在 $m$ 个信道下, 最多有 $m-1$ 个更高优先级的数据流存在插入负载, 因此, 数据流 $F_{k}$ 由于 信道干扰造成的总时延为

$$
\Omega_{k}(\alpha)=\sum_{F_{i} \in \operatorname{hp}\left(F_{k}\right)} I_{k}^{C I}\left(F_{i}, \alpha\right)+U_{k}(\alpha) .
$$

其中, $U_{k}(\alpha)$ 是对所有的 $F_{i} \in \mathrm{hp}\left(F_{k}\right)$ 取 $\min \left(\left|\mathrm{hp}\left(F_{k}\right)\right|, m-1\right)$ 个偏差 $I_{k}^{C I}\left(F_{i}, \alpha\right)-I_{k}^{N C}\left(F_{i}, \alpha\right)$ 的最大值 之和.

当网络包含 $m$ 个信道时, 由于信道竞争造成的时延为

$$
\alpha=\left\lfloor\frac{\Omega_{k}(\alpha)}{m}\right\rfloor+c_{k} .
$$

由信道竞争造成的端到端时延伪上界 $R_{k}^{W H-p s}$ 为 $\alpha$ 的最小值. $\alpha$ 可以通过定点迭代算法 ${ }^{[23]}$ 得 到. 使等式两边相等的 $\alpha$ 值即为 $R_{k}^{W H-p s}$ 的值.

在得到伪上界 $R_{k}^{W H-c h}$ 之后, 下一步再计算由于传输冲突造成的时延, 将其加入到 $R_{k}^{W H-p s}$ 中, 从而得到真正的端到端时延 $R_{k}^{W H}$. 用 $Q(k, i)$ 表示在传输过程中, 数据流 $F_{i}$ 与 $F_{k}$ 共同经过的节点总 数. $\delta_{j}(k, i)$ 表示数据流 $F_{i}$ 与 $F_{k}$ 之间第 $j$ 条最大公用路径上的节点个数, $\delta_{j}^{\prime}(k, i)$ 表示最大共用路径 长度, 最小值为 4 . 由最大共用路径所引起的时延最大为 $3 . \Delta(k, i)$ 表示由 $F_{i}$ 对 $F_{k}$ 引起的传输冲突 造成的端到端时延上界, $\delta$ 表示 $F_{i}$ 和 $F_{k}$ 间的最大共用路径数, 则

$$
\beta=R_{k}^{W H-p s}+\sum_{F_{i} \in \mathrm{hp}\left(F_{k}\right)}\left\lceil\frac{\beta}{t_{i}}\right\rceil \cdot \Delta(k, i)=R_{k}^{W H-p s}+\sum_{F_{i} \in \mathrm{hp}\left(F_{k}\right)}\left\lceil\frac{\beta}{t_{i}}\right\rceil \cdot\left[Q(k, i)-\sum_{j=1}^{\delta}\left(\delta_{j}{ }^{\prime}(k, i)-3\right)\right] .
$$

这样从 $\beta=R_{k}^{W H-p s}$ 开始, 通过定点迭代算法, 求解式 (5) 的最小值就可以得到实际的时延上界 $R_{k}^{W H}$. 


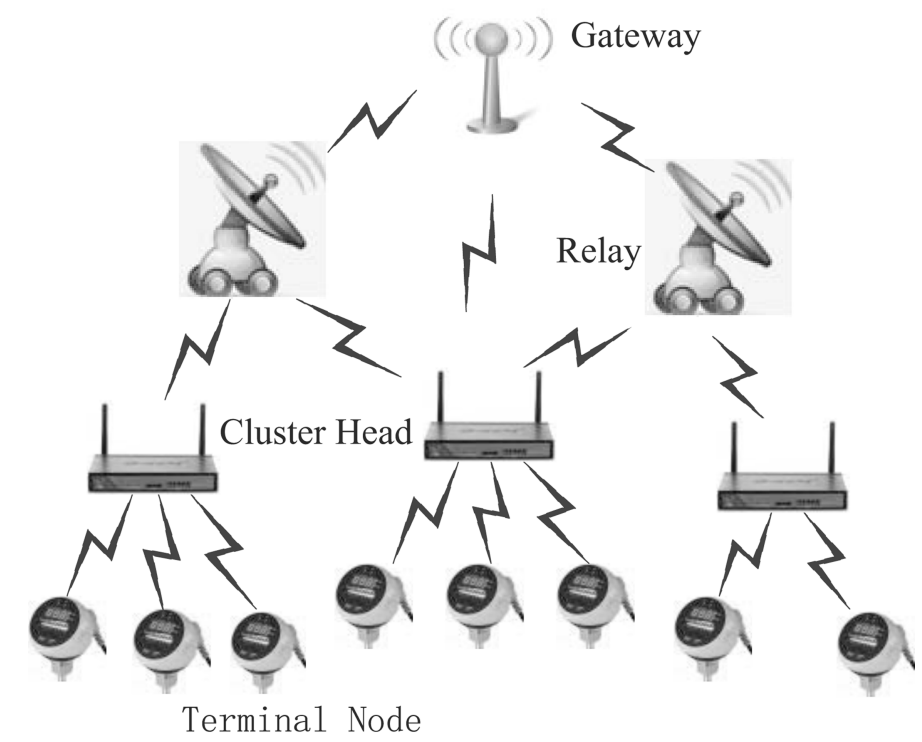

图 2 WIA-PA 的网络结构

Figure 2 The network structure of WIA-PA networks

\subsection{WIA-PA 网络的时延分析}

WIA-PA 为两层拓扑结构, 如图 2 所示. 其下层为星型结构, 由路由设备及终端设备 (固定或手 持) 组成的簇首和簇成员构成. 记网络中簇首的个数为 $n_{W P-C}$, 第 $i$ 个簇首与 $n_{W P-C}^{i}$ 个簇成员连 接. 簇成员经过一跳即可将数据发送给簇首, 发送时隙长度为 $T^{W P-C}$. 簇首配备一个收发器, 每个时 隙只能接收一个簇成员发送的数据, 因此簇成员间发送的时隙必须串行. 网络传输使用的信道总数为 $m^{\mathrm{c}} \cdot h_{W P}$. 不同簇间可以使用不同的信道并行传输. WIA-PA 网络上层为网状结构, 由网关和各簇首 (兼作路由设备) 构成.

WIA-PA 网络分为簇内和簇间两层, WIA-PA 协议中建议网络内通信调度在时间上同样分为两个 阶段, 即簇内调度和簇间调度. 从 WIA-PA 的网络结构可以看出, 其簇间结构和 WirelessHART 网络 相同. 因此可以借助 WirelessHART 网络的分析方法进行时延上界的求解. 在如图 3 所示的网络中, 图 3(a) 表示数据流 $F_{k}$ 在 WirelessHART 网络中的时隙分配, 图 3(b) 表示其在 WIA-PA 网络中的时 隙分配. 由于 WIA-PA 协议对簇间和簇内分别调度的建议, 因此在实际应用中常以相同周期对各节 点数据进行采集, 使得簇内调度能够集中进行, 以增加网络的利用率. 因此在同一个 WIA-PA 网络中 各数据流的 $t_{k}$ 是相同的, 同时该值也是簇内调度的周期. 根据 WIA-PA 的网络结构, 从簇首节点到网 关节点的结构与 WirelessHART 网络的结构相同, 因此从簇首到网关节点的时延上界也为 $R_{k}^{W H}$. 从 终端节点到簇首的时延为常数 $\mathrm{k}$, 如定理 1 所示, 所以在一个 WIA-PA 网络中数据流 $F_{k}$ 的时延为 $R_{k}^{W P}=R_{k}^{W H}+\left\lceil\frac{R_{k}^{W H}}{t_{k}}\right\rceil \cdot k$, 如图 3 所示.

定理1 WIA-PA 网络的簇内传输时延为常数 $k$, 其大小为

$$
k=T^{W P-C} \cdot \max \left\{\max _{\forall i} n_{W P-C}^{i},\left\lceil\frac{\sum_{i=1}^{n_{W P-C}} n_{W P-C}^{i}}{m_{W P}^{\mathrm{c}}}\right\rceil\right\} .
$$

证明 节点所使用的信道总数为 $m_{W P}^{\mathrm{c}} \cdot h$. 当节点的信道间存在干扰时, 为了避免干扰, 使用同一 


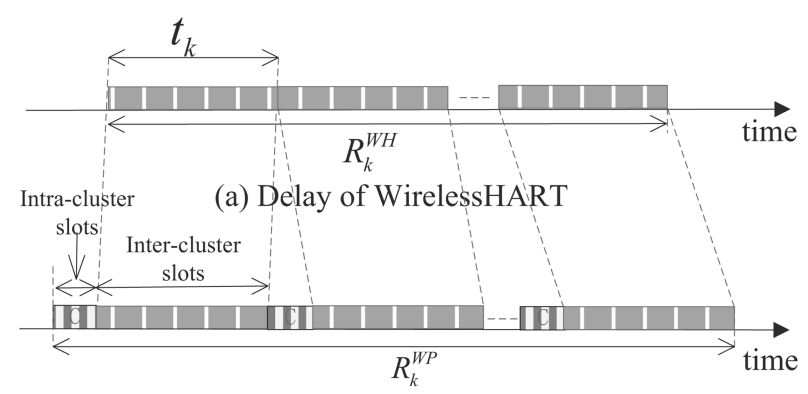

(b) Delay of WIA-PA

图 3 WirelessHART 和 WIA-PA 的时延

Figure 3 The delay of WirelessHART (a) and WIA-PA (b)

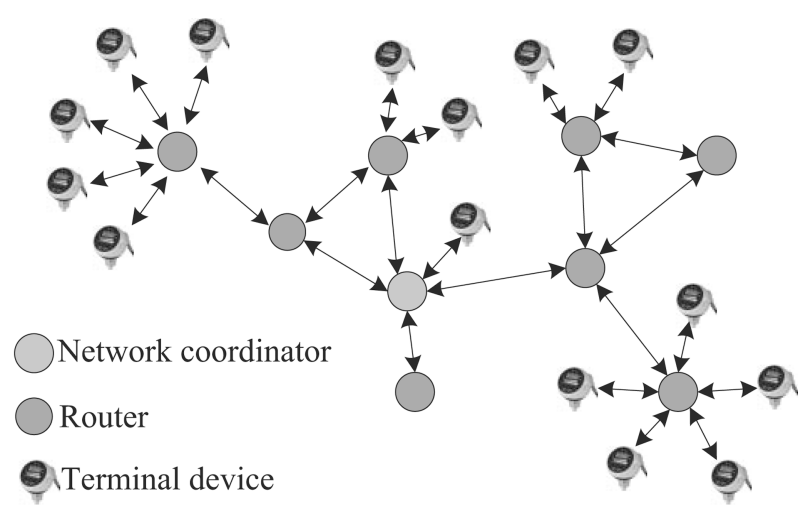

图 4 ZigBee 网络的拓扑结构

Figure 4 The topology of ZigBee networks

信道的节点不能同时发送数据. 这时端到端时延最大值为

$$
T^{W P-C} \cdot\left\lceil\frac{\sum_{i=1}^{n_{W P-C}} n_{W P-C}^{i}}{m_{W P}^{\mathrm{c}}}\right\rceil,
$$

其中 $\sum_{i=1}^{n_{W P-C}} n_{W P-C}^{i}$ 为网络中的终端节点总数. 由于单个簇内所有节点的数据传输必须串行, 所以 端到端时延最大值为 $T^{W P-C} \cdot \max _{\forall i} n_{W P-C}^{i}$. 从而簇内时延的上界为

$$
k=T^{W P-C} \cdot \max \left\{\max _{\forall i} n_{W P-C}^{i},\left\lceil\frac{\sum_{i=1}^{n_{W P-C}} n_{W P-C}^{i}}{m_{W P}^{\mathrm{c}}}\right\rceil\right\} .
$$

\subsection{ZigBee 网络的时延分析}

ZigBee 网络的拓扑结构如图 4 所示, 它采用基于 IEEE 802.15.4 的 CSMA/CAMAC 协议, 帧的发 送形式如图 5 所示. 本文采用文献 [20] 中对 ZigBee 子网部分进行时延分析的方法, 为保证文章的自 完整性, 对分析方法介绍如下. 由于帧的发送采用竞争信道的方式, 因此无法与采用 TDMA 协议的网 络一样进行求解, 但是根据网络的退避指数和退避次数的设置, 仍然可以求出其端到端时延上界.

每个数据包在 ZigBee 网络中经历的整体端到端传输时延由式 (6) 表示:

$$
\text { Delay }=T_{b o}+T_{\text {data }}+T_{a c k}+T_{i f s} .
$$




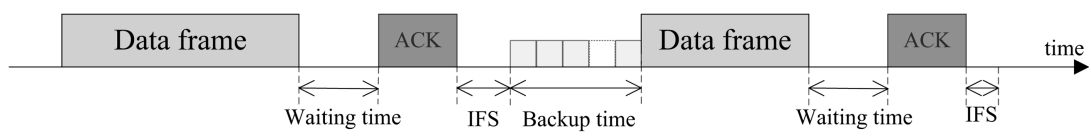

图 5 IEEE802.15.4 的帧发送序列

Figure 5 Frames of IEEE 802.15.4 networks

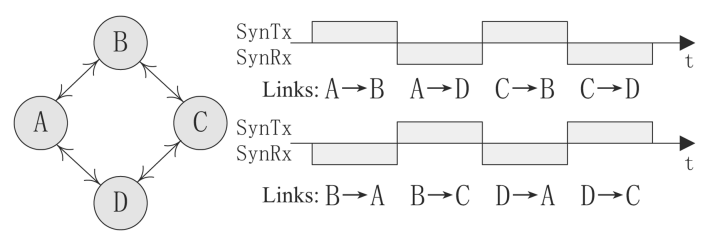

图 $62 \mathrm{P} \mathrm{MAC}$ 协议的状态转换次序

Figure 6 The phase switch method of $2 \mathrm{P}$ MAC

其中 $T_{b o}$ : 退避时间. $T_{d a t a}$ : 数据的传输时间. $T_{a c k}$ : 发送确认时间. $T_{i f s}$ : IFS 帧退避时长.

由于在实际的网络中退避时间比数据的传输时间要大得多, 因此相对于退避时间, 数据的发送时 间可以忽略. 在 CSMA/CA 机制中, 数据包的丢失是由于冲突造成的. 当同时侦听并发现媒质空闲的 节点个数大于 1 时就可能造成冲突. 当不考虑回复确认时, 就认为每个数据包都得以成功发送, 不需 要重传. 设 $B E$ 是退避指数, 在应用中其值一般取 2 . 退避的时隙数是一个随机值, 在 $\left(0,2^{B E}-1\right]$ 区间 内随机取值. 设最大退避次数为 DB, 则到 ZigBee 网络的端到端时延上界为 $R_{k}^{Z B}=\left(2^{B E_{\max }}-1\right) * D B$.

\subsection{2 .11 长距离 WIFI 网络的时延分析}

Raman 等 ${ }^{[3]}$ 提出了 IEEE 802.11 长距离 WIFI 网络的概念, 网络中的每个节点配置大功率单个 或多个的 IEEE 802.11 无线 WIFI 网卡, 采用高增益的定向天线来实现长距离的信号覆盖. 其拓扑相 对固定, 链路距离可达几十甚至几百公里, 可以进行多跳转发来实现源节点和目的节点间的通信. 文 献 [3] 提出了一种专门针对于长距离无线 Mesh 网络的基于 TDMA 的 MAC 层, 称之为 $2 \mathrm{P}$ MAC. 该 协议可以克服长距离 Mesh 网络的问题, 如相邻链路间干扰严重, 链路层重传机制失效等. 其基本思 想是, 在同一时刻, 一个节点上所有的网络接口只能全部同时接收 (SynRx) 或者同时发送 $(\mathrm{SynTx})$ 数 据. 当一个节点从一个状态转换为另一个状态时, 其所有的邻居节点同时也要为相反的状态. 节点的 状态有发送和接收两种, 因此称之为 $2 \mathrm{P}$ MAC (2 Phases MAC).

如图 6 所示为基于 $2 \mathrm{P}$ MAC 协议对于图中的网络结构给出了状态转换顺序, 图中忽略了处理器 时延和传播时延. 线上部分代表发送时隙, 线下部分代表接收时隙. 在初始阶段, 节点 $\mathrm{A}$ 和 $\mathrm{C}$ 为发送 状态, $\mathrm{A}$ 同时向节点 $\mathrm{B}$ 和 $\mathrm{D}$ 发送数据, $\mathrm{C}$ 也同时向 $\mathrm{B}$ 和 $\mathrm{D}$ 发送数据. 在下一时隙, 节点 $\mathrm{A}$ 和 $\mathrm{C}$ 由发 送状态转换为接收状态, 节点 $\mathrm{B}$ 和 $\mathrm{D}$ 由接收状态切换为发送状态, 然后同时向 $\mathrm{A}$ 和 $\mathrm{C}$ 发送数据. 状 态切换不断循环进行. 网络状态转换的周期记为 $T_{L D}$, 其中包括发送阶段的时长 $T_{L D-T x}$ 和接收阶段 的时长 $T_{L D-R x}$, 并有 $T_{L D}=T_{L D-T x}+T_{L D-R x}$. 由该机制可以看出, 此算法要求网络拓扑必须是二 分图, 文献 [3] 证明了通过合理的拓扑生成算法与设置可以保证网络是二分的.

由于 $2 \mathrm{P}$ MAC 协议是基于 TDMA 的, 当采用定向天线且天线间的夹角大于 $30^{\circ}$ 时, 采用正交信 道可以消除干扰. 此时网络的时延为 $d_{k} \times T_{L D}$, 如定理 2 所示. 在工业监控网络中虽然传感节点众多, 但每个节点的数据仅有几个字节且传输周期为秒级, $100 \mathrm{Mbits}$ 的回程网能同时提供几百万个节点的 传输, 所以定理 2 的前提为链路带宽充足. 


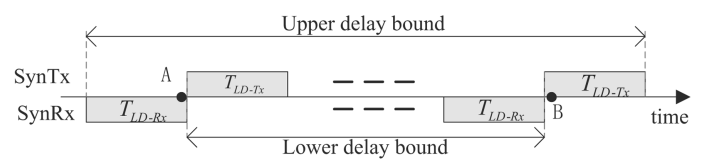

图 7 定理 2 图例

Figure 7 An example of Theorem 2

定理2 当链路带宽充足时, 基于 $2 \mathrm{P}$ MAC 协议的 802.11 长距离 WIFI 网络数据传输的端到端 时延上界为 $R_{k}^{L D}=d_{k} \times T_{L D}$, 下界为 $\left(d_{k}-1\right) \times T_{L D}$.

证明 假设网络的端到端时延大于 $n_{L D} \times T_{L D}$, 由于网络一跳时延一定为 $T_{L D}$, 若时延大于 $n_{L D} \times T_{L D}$, 则说明数据在传输过程中在某点停留时间至少为 $2 \times T_{L D}$. 由于网络可以划分为二分图, 因此每个节点可以连续的在接收和发送状态间循环的切换, 并且网络带宽充足, 所以当数据在 $T_{L D-T x}$ 时段到达时, 必定连续在下一个 $T_{L D-T x}$ 时段被传输. 综上数据在某点停留的时间至多为 $T_{L D}$, 假设 与此矛盾. 因此网络的端到端时延上界为 $d_{k} \times T_{L D}$.

数据的最差时延 (即上界时延 $R_{k}^{L D}$ ) 情况如图 7 所示, 一个数据包在第一跳的 $T_{L D-R x}$ 开始处到 达, 在最后一跳的 $T_{L D-T x}$ 结束时间被传送到目的节点. 而在实际网络中一个数据包最晚的到达时间 为 $T_{L D-R x}$ 的结束点 $\mathrm{A}$, 最早的送达时间处于 $T_{L D-T x}$ 的开始时间 $\mathrm{B}$ 点, 因此时延下界为 $n_{L D} \cdot T_{L D}-$ $T_{L D-R x}-T_{L D-T x}=n_{L D} \cdot T_{L D}-T_{L D}=\left(d_{k}-1\right) \cdot T_{L D}$.

\section{5 广域异构网络整体端到端时延分析}

由于每个子网中传感器节点数量多, 但每个节点的数据量少, 因此要对子网数据进行数据聚合后 传输. 从各个子网采集的数据经过子网传输以后, 在 IEEE 802.11 长距离 WIFI 与子网连接的网关节 点处进行聚合, 由于聚合周期为 $T_{a g}$, 因此聚合引起的时延最大为 $R_{a g}=T_{a g}$. 经过数据聚合以后, 数据再经过 IEEE 802.11 长距离 WIFI 回程网传送到数据中心, 从而数据在广域异构网络中由终端 节点到数据中心的端到端时延为在子网中的时延、聚合时延及在 WIFI 回程网中的时延三者加和, 即 $R_{k}=R_{k}^{L D}+R_{k}^{x}+R_{a g}$, 根据上面的分析, 分别计算如下.

- 在 802.11 长距离 WIFI 和 WirelessHART 组成的网络中, 时延上界为 $R_{k}=n_{L D} \cdot T_{L D}+R_{k}^{W H}+$ $R_{a g}$, 其中 $R_{k}^{W H}$ 由式 (5) 求得.

- 在 802.11 长距离 WIFI 和 WIA-PA 组成的网络中, 时延上界为 $R_{k}=n_{L D} \cdot T_{L D}+R_{k}^{W P}+R_{a g}=$ $R_{k}^{W P}=n_{L D} \cdot T_{L D}+R_{k}^{W H}+\left\lceil\frac{R_{k}^{W H}}{t_{k}}\right\rceil \cdot k+R_{a g}$, 其中

$$
k=T_{i}^{W P-C} \cdot \max \left\{\max _{\forall i} n_{W P-C}^{i},\left\lceil\frac{\sum_{i=1}^{n_{W P-C}} n_{W P-C}^{i}}{m_{W P}^{\mathrm{c}}}\right\rceil\right\} .
$$

- 在 802.11 长距离 WIFI 和 ZigBee 组成的网络中, 时延上界为 $R_{k}=n_{L D} \cdot T_{L D}+R_{k}^{Z B}+R_{a g}=$ $n_{L D} \cdot T_{L D}+\left(2^{B E_{\max }}-1\right) \cdot D B+R_{a g}$.

\section{5 实验与结果分析}

本实验将本文所提出的分析方法与仿真、半实物仿真方法分别进行对比. 评价指标采用悲观率 (pessimism ratio), 悲观率的计算方法是取理论分析的网络端到端时延与仿真 (或半实物仿真) 得到的 网络端到端时延的比值. 


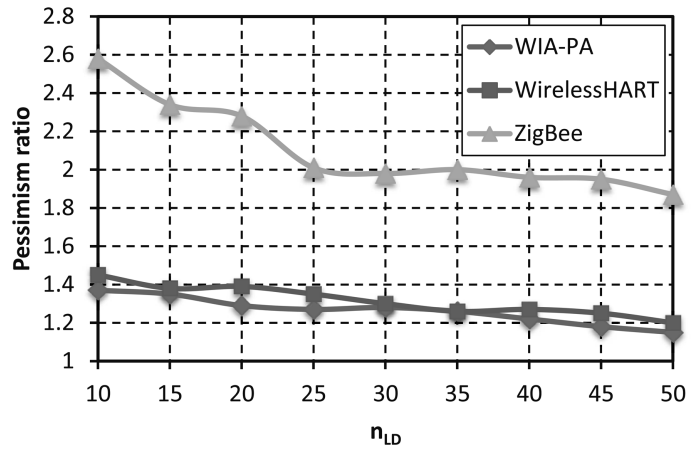

图 8 悲观率与长距离 WIFI 回程网节点数的关系

Figure 8 The relationship of pessimism ratio and the number of LD-WIFI nodes

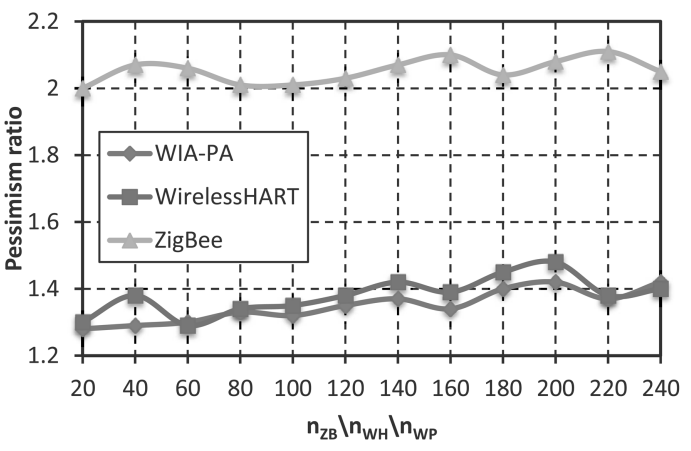

图 9 悲观率与子网网络规模的关系

Figure 9 The relationship of pessimism ratio and subnetwork scale

\section{1 仿真实验}

本小节将分析结果与仿真模拟结果进行对比, 以悲观率作为评价指标. 通过对问题的分析可知, 802.11 长距离 WIFI 回程网的节点数 $n_{L D} 、 \mathrm{MAC}$ 协议时间槽的长度 $T_{L D}$ 、各子网的节点数 $\left(n_{W H}, n_{W P}\right.$, $\left.n_{Z B}\right)$ 以及网关节点的聚合周期 $T_{a g}$ 都会对分析结果产生影响. 因此本文分别变化以上 4 个参数得到 网络在不同配置情况下的端到端时延变化曲线, 用以说明所提出的分析方法在不同配置下的分析效果.

本实验采用 $\mathrm{C}++$ 实现仿真. 网络基本设置如下: 随机生成 25 个节点的 802.11 长距离 WIFI 回 程网拓扑, 并分别生成节点规模为 100 的 WirelessHART, ZigBee 和 WIA-PA 子网拓扑. 其中, ZigBee 网络的每个中继器及 WIA-PA 网络的每个簇首节点所负责的节点个数在 3 到 5 个之间. 网关节点处 数据的聚合时间周期为 $10 \mathrm{~ms}, 802.11$ 长距离 WIFI 的 MAC 时隙长度为 $10 \mathrm{~ms}$.

图 8 11 为改变 4 个参数中的某一个而得到的悲观率变化趋势图. 图中图例项 "WirelessHART" (“WIA-PA” 或 “ZigBee”) 表示数据在子网 WirelessHART (WIA-PA 或 ZigBee ) 和回程网间传输时延 的悲观率.

图 8 是随着 802.11 长距离 WIFI 回程网节点总数的变化, 悲观率的变化曲线. 根据定理 2 , 当转 换时间和跳数一定时, 802.11 长距离 WIFI 回程网的最大悲观率为 $\frac{d_{k}}{d_{k}-1}$. 随着 802.11 长距离 WIFI 回 程网节点数增多, 跳数增多, 回程网的悲观率将变小, 因此网络整体的悲观率随着 802.11 长距离 WIFI 回程网节点数的增加呈现下降趋势.

图 9 是随着子网网络规模的增大, 悲观率的变化曲线. 由于随着网络规模的增大, 按照最差情况 进行时延估计会造成估计误差的增大, 因此悲观率整体呈现上升趋势.

图 10 是随着网关节点聚合周期的增大, 悲观率的变化曲线. 本文提出的分析方法在每个数据包 的时延中加入固定的聚合周期, 但实际网络中数据到达子网网关节点等待聚合的时间一定小于聚合周 期, 因此聚合周期越大, 本方法加入到圥余时延越长, 悲观率越高.

图 11 是随着 802.11 长距离 WIFI MAC 协议时间槽长度的增大, 悲观率的变化曲线. 由于 802.11 长距离 WIFI 时间槽长度越大, 则其造成的时延在端到端时延中的比重越大. 而 802.11 长距离 WIFI 的悲观率最差值与跳数有关. 当 802.11 长距离 WIFI 的时间槽增大到 $90 \mathrm{~ms}$ 之后, 其他部分的时延对 网络整体时延影响减小, 网络的悲观率近似等于 $\frac{d_{k}}{d_{k}-1}$.

另外, 在以上实验中 WirelessHART 与 WIA-PA 相比悲观率较高是因为同样节点数量的两种网 络, WIA-PA 中簇内节点的时延为定值, 因此减小了一部分悲观率. 这也说明 WIA-PA 的两层结构既 


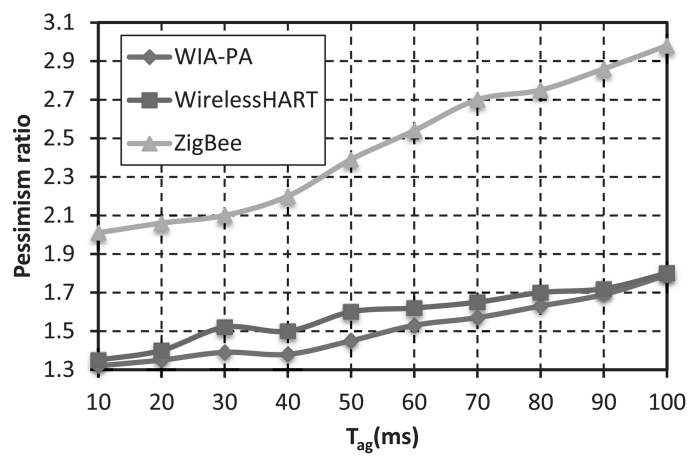

图 10 悲观率与网关节点聚合周期的关系

Figure 10 The relationship of pessimism ratio and data aggregation period of the gateway

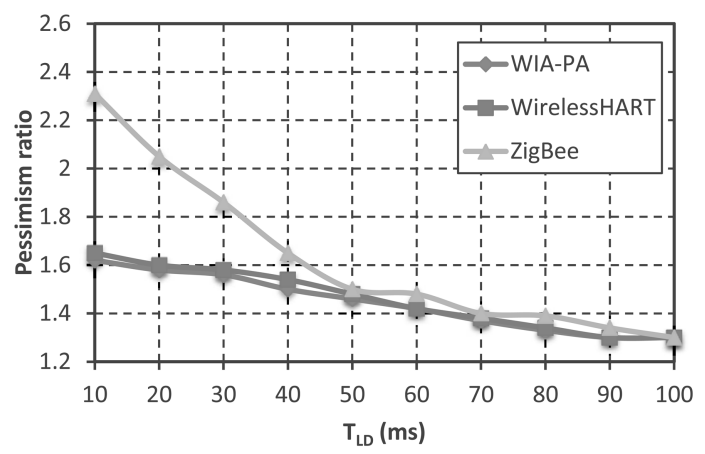

图 11 悲观率与长距离 WIFI MAC 协议时间槽长度 的关系

Figure 11 The relationship of pessimism ratio and time slots of the LD-WIFI MAC protocol

减小了网状拓扑传输时延的不确定性, 又可以具有网状结构节点部署灵活与多路径抗干扰能力等优 点. 由于 ZigBee 网络采用退避的方式接入信道, 本分析方法为了得到最差的端到端时延, 将最大退避 时间和次数的乘积作为 ZigBee 子网的传输时延. 因此与其他网络相比, 其整体的误差较大, 悲观率较 高. ZigBee 由于其低功耗的特定被广泛用于传感网络, 但并非针对时延敏感的工业监控网络而设计, 此种网络由于其基于冲突的链路接入机制导致其端到端时延抖动较大. 而 WirelessHART 和 WIA-PA 针对工业系统设计, 采用基于 TDMA 的链路接入机制, 能够为传输提供可靠的时延保障. 本实验结果 与 3 种网络特性完全契合.

\section{2 半实物系统实验}

Qualnet/Exata 是美国 Scalable Networks Technologies 公司针对新型无线通信技术而设计开发的 网络仿真系统, 可以与真实网络中的设备进行通信, 其精确程度能够与真实网络相謧美. 由于在现有 的条件下, 大规模部署长距离 WIFI 比较困难, 因此实验中以开发的 WIA-PA 节点和 802.11 无线交 换机组成的物理传输平台为基础, 利用 Qualnet/Exata 仿真软件搭建了一套半实物仿真平台. 平台中 WIA-PA 节点和交换机采用硬件实现. IEEE 802.11 长距离 WIFI 回程网中的部分路由节点采用硬件 实现, 其他都为虚拟节点. 仿真主机采用 8 核、 $4 \mathrm{~GB}$ 内存的计算机, 数据中心采用 24 核、2TB 内存 的服务器实现. 实验中根据工业无线网络的实际应用环境, 将网络的整体架构设置为如图 12 所示的 情景.

实验得到数据通过 WIA-PA 网络到数据中心的端到端时延悲观率平均值为 1.2 , 通过 WirelessHART 网络到数据中心的端到端时延悲观率平均值为 1.6, 通过 ZigBee 网络到数据中心的端到端时延 悲观率的平均值为 2.0. 根据文献 [15] 和 [24] 的实验与分析结果可知, 在悲观率分析方面, 现有的无线 多跳网络的悲观率都在 2 左右, 最优情况下可达到 1.6. 而本文考虑在多种网络组成的异构网络中的 情况, 问题更为复杂. 因此, 根据实验结果, 可以验证本文分析方法的有效性.

\section{6 结论}

在工业无线监控应用中, 网络的异构性使数据传输的端到端时延分析变得困难. 现有的分析都基 于单一网络模型或非工业异构网络, 没有对异构的工业控制网络的端到端时延进行系统的研究. 本文 


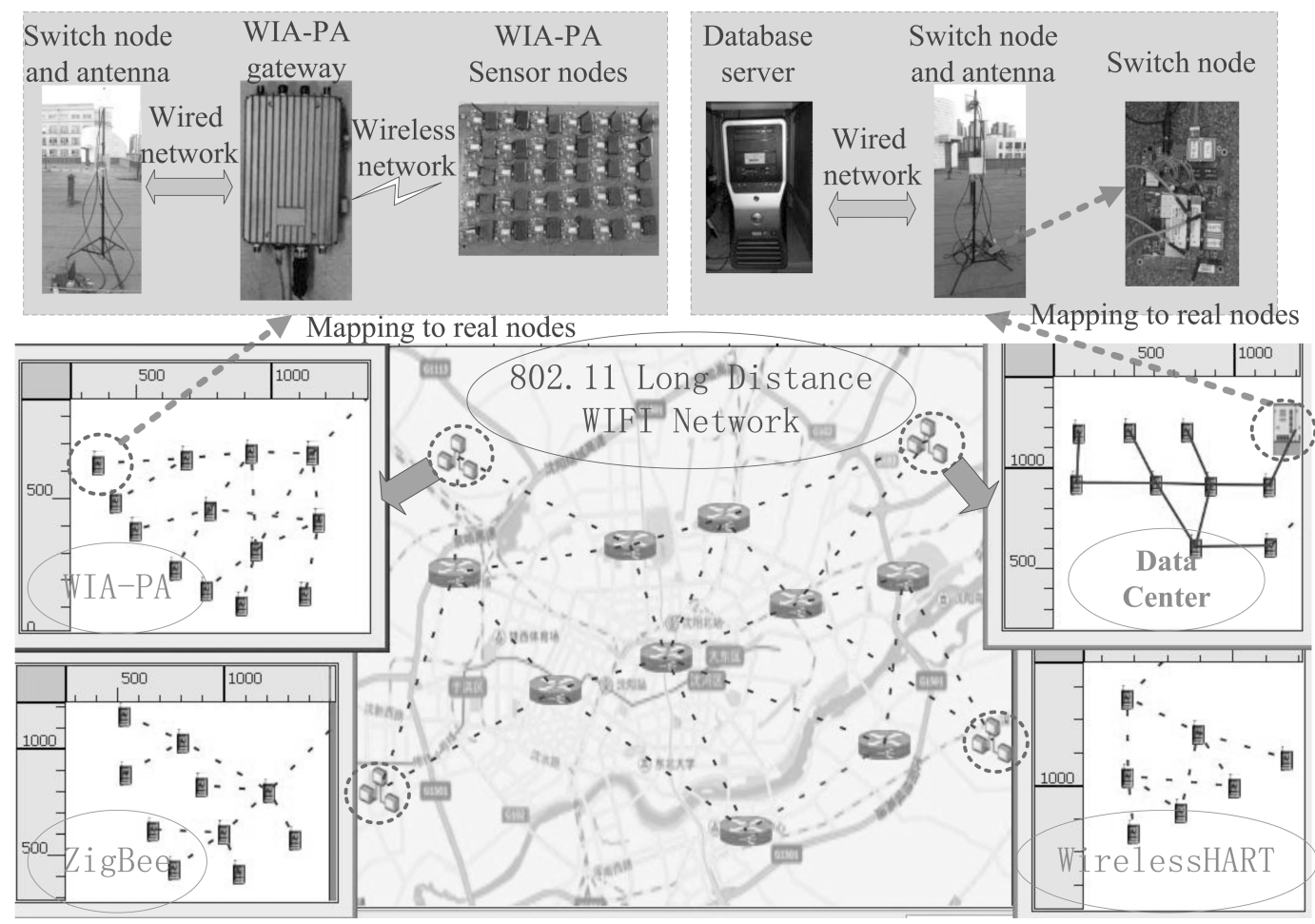

图 12 半实物仿真平台

Figure 12 The semi-physical simulation platform

分析了在以 802.11 长距离 WIFI 为回程网, 以 WirelessHART, ZigBee 和 WIA-PA 为子网的异构网络 中数据的端到端时延, 为网络设计者提供一种验证网络实时性的有效方法. 本文的实验部分分别通过 仿真与半实物仿真的方式进行, 实验结果说明了分析方法的有效性.

\section{参考文献}

1 Zeng P, Yu H B. Industrial wireless network WIA standard system and key technologies. Autom Panorama, 2009, 26: 24-27 [曾鹏, 于海斌. 工业无线网络 WIA 标准体系与关键技术. 自动化博览, 2009, 26: 24-27]

2 Rui W Z, Jiang H H, Hou C Y. Survey and prospect of networked control system. Inform Control, 2012, 41: 83-88 [茌万智, 江汉红, 侯重远. 网络控制系统研究综述与展望. 信息与控制, 2012, 41: 83-88]

3 Raman B, Chebrolu K. Design and evaluation of a new MAC protocol for long-distance 802.11 mesh networks. In: Proceedings of the 11th Annual International Conference on Mobile Computing and Networking. Cologne: ACM, 2005. 156-169

4 Liang W, Zhang X L, Xiao Y, et al. Survey and experiments of WIA-PA specification of industrial wireless network. Wirel Commun Mob Comput, 2011, 11: 1197-1212

5 Soares S. Integrating data governance and big data with business processes. IBM Data Magazine. http://www.zigbee.org/zigbee-for-developers/zigbee3-0/. 2012

6 Gao Z L, Liang B J. Digital Oilfield in China Technology and Development of Network Oilfield. Beijing: Science Press, 2013. 117-136 [高志亮, 梁宝娟. 数字油田在中国 —— 油田物联网技术与进展. 北京: 科学出版社, 2013. 117-136]

7 Scharbarg J L, Boyer M, Fraboul C. Interconnecting can busses via an ethernet backbone. Fieldbus Syst their Appl, 2005, 6: 206-213

8 Shang Z J, Cui S J, Wang Q S. Network calculus based dimensioning for industrial wireless mesh networks. Appl Mech Mater, 2013, 303: 1989-1995 
9 Wang K, Ciucu F, Lin C, et al. A stochastic power network calculus for integrating renewable energy sources into the power grid. IEEE J Sel Areas Commun, 2012, 30: 1037-1048

10 Hua Y, Liu X. Scheduling design and analysis for end-to-end heterogeneous flows in an avionics network. INFOCOM, 2011 Proceedings IEEE. Shanghai: IEEE, 2011. 2417-2425

11 Li X, Scharbarg J L, Fraboul C. Analysis of the pessimism of the trajectory approach for upper bounding end-to-end delay of sporadic flows sharing a switched Ethernet network. In: Proceedings of RTNS, Nantes, 2011. 149-158

12 Kemayo G, Ridouard F, Bauer H, et al. Optimism due to serialization in the trajectory approach for switched Ethernet networks. Proc Int Conf Junior Res Workshop Real-Time Comput (JRWRTC), Sophia Antipolis, 2013. 13-16

13 Medlej S. Scalable Trajectory Approach for ensuring deterministic guarantees in large networks. Paris: Universit é Paris-Sud, 2013

14 Li X, Scharbarg J, Fraboul C. Worst-case delay analysis on a real-time heterogeneous network. In: 2012 7th IEEE International Symposium on Industrial Embedded Systems (SIES). Karlsruhe: IEEE, 2012. 11-20

15 Saifullah A, Xu Y, Lu C Y, et al. End-to-end delay analysis for fixed priority scheduling in WirelessHART networks. In: Proceedings of the 17th IEEE Real-Time and Embedded Technology and Applications Symposium. Chicago: IEEE, 2011. 13-22

16 Saifullah A, Xu Y, Lu C Y, et al. End-to-End communication delay analysis in wirelessHART networks. Technical Report WUCSE-2011-86. St Louis: Washington University, 2011

17 Jurcik P, Severino R, Koubaa A, et al. Real-time communications over cluster-tree sensor networks with mobile sink behaviour. In: Proceedings of the 14th IEEE International Conference on Embedded and Real-Time Computing Systems and Applications. Taiwan: IEEE, 2008. 401-412

18 Schmitt J B, Roedig U. Sensor network calculus-a framework for worst case analysis. In: Proceedings of the 1st IEEE International Conference on Distributed Computing in Sensor Systems. Berlin: Springer, 2005. 141-154

19 Chen F, Talanis T, German R, et al. Real-time enabled IEEE 802.15.4 sensor networks in industrial automation. In: Proceedings of the IEEE International Symposium on Industrial Embedded Systems. Lausanne: IEEE, 2009. 136-139

20 Yaqoob M M, Israr I, Javaid N, et al. Transmission delay of multi-hop heterogeneous networks for medical applications. arXiv, 2012: 1208.2409

21 Zhong T, Mengjin C, Peng Z, et al. Real-time communication in WIA-PA industrial wireless networks. In: Proceedings of the 3rd IEEE International Conference on Computer Science and Information Technology. Chengdu: IEEE, 2010. $600-605$

22 Zhang X, Liang W, Zheng M, et al. Distributed and dynamic TDMA channel scheduling algorithm for WIA-PA. In: Proceedings of the IEEE International Conference on Intelligent Computing and Intelligent Systems. Shanghai: IEEE, 2009. 462-466

23 Joseph M, Pandya P. Finding response times in a real-time system. Comput J, 1986, 29: 390-395

$24 \mathrm{Wu}$ C, Sha M, Gunatilaka D, et al. Analysis of EDF scheduling for wireless sensor-actuator networks. IEEE/ACM Symposium on Quality of Service (IWQoS'14). Hong Kong: IEEE, 2014

\title{
End-to-end delay analysis in wide-area heterogeneous wireless network for industrial monitoring and control applications
}

\author{
WANG JinTao ${ }^{1,2}$, JIN Xi $^{1}$, ZENG Peng ${ }^{1 *} \&$ LI Dong ${ }^{1,2}$ \\ 1 Key Laboratory of Networked Control Systems, Shenyang Institute of Automation, Chinese Academy of Sciences, \\ Shenyang 110016, China; \\ 2 University of Chinese Academy of Sciences, Beijing 100049, China \\ *E-mail: zp@sia.cn
}

Abstract With the development of wireless communication, the wireless communication technologies based on WirelessHART, WIA-PA, and ZigBee are used widely in the industrial control field. However, owing to the con- 
straint of capital, technology, equipment, etc., diverse network technologies have long coexisted. Delay analysis is an effective method for detecting real-time network properties in the network design phase. Currently, there is no particular method of analyzing end-to-end transmission delay in wide-area industrial heterogeneous networks. Consequently, based on wide-area heterogeneous backhaul networks with industrial control applications, end-toend delay in wide-area heterogeneous environments, including IEEE 802.11 long-distance WIFI, WirelessHART, ZigBee, and WIA-PA, is analyzed theoretically and verified experimentally. These comparative experiments show that, when the node number of an 802.11 long-distance WIFI backhaul network is greater than 25 , and the data aggregation period of the gateway is less than $30 \mathrm{~ms}$, the pessimism ratio is guaranteed to be within 2.3 in the network for ZigBee and within 1.5 for others. The rationale and validity of the method are proved.

Keywords industrial monitoring and control, heterogeneous network, wireless network, end-to-end delay, analysis

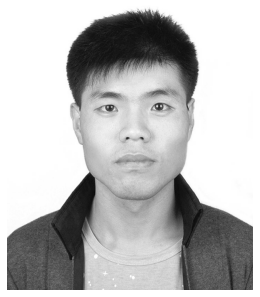

WANG JinTao is a Ph.D. student at the University of the Chinese Academy of Sciences at the Shenyang Institute of Automation, Chinese Academy of Sciences. His research interests include wireless MESH networks, communication scheduling, and QoS guarantees.

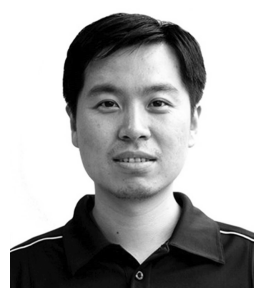

ZENG Peng is Professor at the Shenyang Institute of Automation, Chinese Academy of Sciences. His research has involved industrial communication and wireless sensor networks.

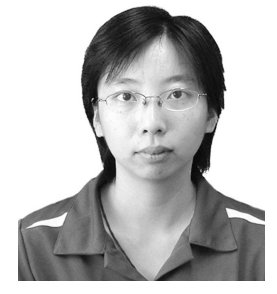

JIN $\mathbf{X i}$ is a research associate at the Shenyang Institute of Automation, Chinese Academy of Sciences. Her research interests include communication scheduling, energy-harvesting sensor networks, and real-time systems.

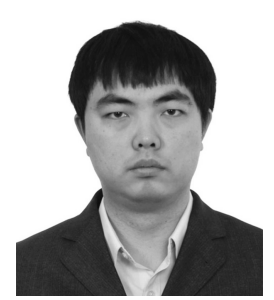

LI Dong is a Ph.D. student at the University of the Chinese Academy of Sciences at the Shenyang Institute of Automation, Chinese Academy of Sciences. His research interests include wireless communication, time synchronization, industrial communication, and wireless sensor networks. 